\title{
25 Research Square \\ Study on the morphology of Mature ovarian follicles using Image Processing Toolbox
}

\section{LI-Feng Bao}

Huaian College of Information Technology https://orcid.org/0000-0002-8724-0271

Hui-Mei Li ( $\nabla$ doctorhuimeili@163.com )

https://orcid.org/0000-0003-2608-0283

Research article

Keywords: follicle, shape measurement, ovulation day

Posted Date: July 21st, 2020

DOI: https://doi.org/10.21203/rs.3.rs-42445/v1

License: (c) (i) This work is licensed under a Creative Commons Attribution 4.0 International License.

Read Full License 


\section{Abstract}

Objective: This study aims to investigate the morphological characteristics of mature follicles with clinical value.

Study Design:a retrospective study

Methods: The five morphological indexes of follicles in 72 natural ovulation cycles were measured. The shoot time of follicle photos was the day before ovulation. Measurement software was the MATLAB Image Processing Toolbox.

Results: The measured average area, perimeter, equivalent circle diameter and roundness were $301.29 \pm$ $8.52 \mathrm{~mm}^{2}, 62.23 \pm 6.82 \mathrm{~mm}, 20.21 \pm 3.08 \mathrm{~mm}$ and $0.89 \pm 0.05$, respectively; and the maximal diameter was $23.12 \pm 3.06 \mathrm{~mm}$.

Conclusion: Image Processing Toolbox is a simple and convenient measurement software. The standardization of the reference indexes of follicular morphology has an important clinical application value in reproductive medicine.

Research Highlights and Graphical Abstract: In the present study, the morphological characteristics of these folliclesinclude the area, perimeter, maximal diameter, equivalent circle diameter and roundness were preliminarily studied using the related measurement software.

\section{Introduction}

Monitoring the growth and maturation of follicles by B ultrasound [1-4] has been widely used in the diagnosis and treatment of infertility. The morphological quality of the follicles [5-7] largely reflects the quality of the ova, is closely associated with pregnancy rate and delivery rate, and even affects the incidence of fetal malformation. However, there is no quantitative standard for the morphological characteristics of mature follicles in the domestic academic community. In the clinical diagnosis and treatment, a consensus has been achieved in only one index: the average diameter of mature follicles [1].

From the morphological perspective, the morphological indexes that reflect the quality of follicle include the area, perimeter, maximal diameter, equivalent circle diameter and roundness. In the present study, a certain number of follicular follicles were collected, and the morphological characteristics of these follicles were preliminarily studied using the related measurement software.

\section{Materials And Methods}

\subsection{Materials}

The source of the 72 samples used in this study was those right age women who established the birth service cards in the reproductive center of Huai'an First People's Hospital and became pregnant and 
delivered from 2015 to 2017. After obtained an informed consent from these women, the natural ovulation cycle during pregnancy was monitored by $\mathrm{B}$ ultrasound, and pictures of the follicles on day - 1 (the day before ovulation) were taken.

\subsection{Morphological measurement software}

In this study, we used the Japanese ALOKASSD-1400 ultrasound diagnostic apparatus, 3.5-MHz convex array probe and 5.0-MHz transvaginal probe. Follicle photos were taken using 8-million pixel SONYSDSCT20 digital camera.

The MATLAB Image Processing Toolbox was used to measure the morphological parameters of follicles. MATALB is one of a series of products of the MathWorks company, and Image Processing Toolbox is an image processing toolbox in MATALB. It can be applied in image acquisition [8,9] analysis [10-12] development of visualization and algorithm [13] and other services. Compared with traditional programming languages (such as C, C++ and Fortran), Image Processing Toolbox make solving the problem of technical computing more easy.

MATALB has very low hardware and software requirements, its written programs can run in a stand-alone window system. Clinicians can obtain the measurement results of morphological indicators by simply using the graphical interface of MATALB.

\subsection{Methods for quantitative determination of morphology}

In this study, all of the follicle photos were taken at day -1 of the natural ovulation cycle in which the woman successfully conceived. Ultrasound images acquired on the ovulation date (day 0 ) showed that the dominant follicle disappeared. In addition to B ultrasound imaging features, the determination of ovulation day should also be with reference to basal body temperature (BBT), cervical score (CS) $[14,15]$ and urine LH test paper, in order to ensure the accuracy of time determining [4].

The program written in MATALB Image Processing Toolbox [3] was used to quantitatively determine the morphology of each follicle on the photos. The determined morphological indexes included the area, perimeter, maximal diameter, equivalent circle diameter and roundness. The formula for calculating the degree of roundness of follicles is ( $\mathrm{A}$ is the follicular area, $\mathrm{P}$ is the follicular perimeter). After the completion of all the measurement work, a total of 72 samples were obtained.

\subsection{Statistical methods}

The measurement data were analyzed using statistical software SPSS12.0, were expressed as mean \pm standard deviation $( \pm S D)$. The mean data were compared using $t$-test, and count data were compared using $X^{2}$ test.

\section{Results}

The average values of the parameters of the 72 samples are shown in Table 1. The five morphological indexes of mature follicles were the follows: the average area was $301.29 \pm 8.52 \mathrm{~mm}^{2}$, average perimeter 
was $62.23 \pm 6.82 \mathrm{~mm}$, maximal diameter was $23.12 \pm 3.06 \mathrm{~mm}$, average equivalent circle diameter was $20.21 \pm 3.08 \mathrm{~mm}$, and average roundness was $0.89 \pm 0.05$.

Table 1

Follicle morphology measurement

\begin{tabular}{|lll|}
\hline morphological index & unit & Value \\
\hline sample number & & 72 \\
\hline area & $\mathrm{mm}^{2}$ & $301.29 \pm 8.52$ \\
\hline perimeter & $\mathrm{mm}$ & $62.23 \pm 6.82$ \\
\hline maximum diameter & $\mathrm{mm}$ & $23.12 \pm 3.06$ \\
\hline equivalent circle diameter & $\mathrm{mm}$ & $20.21 \pm 3.08$ \\
\hline roundness & & $0.89 \pm 0.05$ \\
\hline
\end{tabular}

The $95 \%$ confidence level $(x \pm 1.96 \mathrm{SD})$ of each parameter listed in Table 1 are within the range of medical reference value.

\section{Discussion}

\subsection{Determination of sampling time of follicles in nature ovulation cycle}

In a natural ovulation cycle, the growth and development of ovarian follicles are dynamic. A follicle produced in a natural cycle grows and develops at a certain speed, its morphology is different on every day during the cycle. In this study, the shoot times of photos of all samples were assigned on the day before ovulation in the natural ovulation cycle, in accordance with the requirements of statistical mathematics to sample selection. To accurately determine day -1 , in addition to ultrasound image characteristics, other common monitoring methods, such as BBT, CS, LH, etc., are also required to use

\subsection{Two aspects to reflect follicular quality}

When monitoring follicle using B ultrasound, although there is no definite quantization standard for determining follicular quality in clinic, an empirical consensus has been achieved. It includes two aspects: external morphological characteristics and internal turbidity degree of follicles. This study preliminary discussed the former aspect.

\subsection{Prospect and future}

Human natural fertility is mainly single birth. A follicle is produced in a natural ovulation cycle in a women at childbearing age, then grows and develops to mature. A healthy follicle is fertilized after ovulation and grows until delivery. Morphological characteristics of follicle in the cycle is related to pregnancy, childbirth, and even fetal defect and deformity rate. But the understanding of this correlation is only in the level of empirical consensus. Its quantitative research is a complex systemic engineering, needs a number of large samples and long-term follow-ups. This study is just a small step for this 
systemic engineering.

In the field of diagnosis and treatment of infertility, individuation of ovulation induction treatment, that is, developing the optimal option of medication according to the specific situations of every infertility patients, has become the basic consensus of the clinical diagnosis and treatment. dynamic observation on the growth morphology of follicle in ovulation induction cycle of patients through B ultrasound monitoring is the basic auxiliary means of ovulation treatment, clinicians can determine the effect of the used ovulation-inducting drugs according to the B ultrasound images of ovarian follicles of patients, and adjust the medication in time. At present, however, this step is also just in an empirical level, that is, clinicians determine the advantages and disadvantages of the morphological quality of ovarian follicles based on their experience, lacking relatively unified and scientific quantitative standard. This study made a preliminary attempt in this area.

\subsection{Shortcomings}

In addition to the sample size is not particularly large, the samples were all only followed up to the smooth delivery of the volunteers, where no obvious defect was observed in the fetal appearance. Then day -1 in its natural pregnancy cycle was deducted forward. The selected samples obviously could not exclude the possibility that fetus had recessive defects. Therefore, more adequate studies not only need more samples, also requires long-term follow-ups.

\section{Declarations}

\section{Ethics approval and consent to participate}

This study was conducted in accordance with the declaration of Helsinki.

This study was conducted with approval from the Ethics Committee of Huaian First People's Hospital with Nanjing Medical University.

Written informed consent was obtained from all participants.

\section{Competing Interest}

The authors declare that they have no competing interests.

Author's Contribution Li-Feng Bao and Hui-Mei Li carried out the study .Xu Min drafted the manuscript.LiFeng Bao and Hui-Mei Li participated in the design of the study. Hui-Mei Li conceived of the study, and participated in its design and helped to draft the manuscript. All authors read and approved the final manuscript.

\section{Abbreviations}


BBT basal body temperature

CS cervical score

\section{References}

[1] Lenz S, et al. Significance of the ultrasonic morphology of preovulatory ovarian follicles prior to in vitro fertilization. . IActa Europaea fertilitatis, 1983;14 (5), pp.305-10.

[2] Baerwald AR,et al..Characterization of ovarian follicular wave dynamics in women. Biol Reprod.2003;69(3):1023-31. .

[3] Goodman AL, et al. Follicle dominance and ovarian asymmetry aftar luteectomy in rhesuh monkeys. Am J Physiol.1982;243(4):E325-31.

[4] Tulandi T, et al.. Ovulation induction by human menopausal gonadotropin with ultrasonic monitoring of the ovarian follicles. . International journal of fertility 1987;32 (4), pp.312-5.

[5] Stouffer Richard, et al. Overriding follicle selection in controlled ovarian stimulation protocols: Quality vs quantity . Reproductive Biology and Endocrinology 2004;2:32.

[6] Zeleznik Anthony. The physiology of follicle selection . Reproductive Biology and Endocrinology, 2004, 2:31.

[7] Padhy Nabaneeta, et al. Antral follicle size in the downregulated cycle and its relation to in vitro fertilization outcome. Journal of Human Reproductive Sciences. 2009, 2(2):68-71.

[8] Haralick R M,et al. SURVEY:Image Segmentation Techniques Computer Vision,Graphics,and Image Processing 1985, 29(1):100-132.

[9] Pal N R,et al. A review on Image Segmentation Techniques Pattern Recognition 1993;26(9):1277-1294.

[10] P K Sahoo,et al..Survey of thresholding Techniques Computer Vision,

Graphics,and Image Processing. 1988,41(2):233-260.

[11] Bhabatosh Chanda,et al Multi-Scale Morphologic Edge Detector Pattern Recognition 1998;31(10):1469-1478.

[12] Philippe Schmid,.Segmentation of Digitized Dermatoscopic Images by Two-Dimensional Color Clustering IEEE Trans Neural networks.1999,18(2):164-171.

[13] Tian B,et al. A study of cloud classification with neural networks using spectral and textural features IEEE Trans Neural networks.1999;10(1):138-151. 
[14] Templeton A A,et al. Relation between the luteinizing hormone peak, the nadir of the basal body temperature and the cervical mucus score British Journal of Obstetrics and Gynaecology 1982;89 (12), pp.985-8.

[15] Leader A,et al. The prediction of ovulation: a comparison of the basal body temperature graph, cervical mucus score, and real-time pelvic ultrasonography Fertility and Sterility 1985;43 (3), pp.385-8.

\section{Supplementary Files}

This is a list of supplementary files associated with this preprint. Click to download.

- follicleimage.JPG 